\title{
Introduction to Human- and Task-Centered Assistance Systems Minitrack
}

\author{
Jens P. Wulfsberg \\ Helmut-Schmidt-University \\ Hamburg \\ Germany mwulf@hsu-hh.de
}

\author{
Tobias Redlich \\ Helmut-Schmidt-University \\ Hamburg \\ tobias.redlich@hsu-hh.de
}

\author{
Robert Weidner \\ Helmut-Schmidt-University \\ Hamburg, Germany \\ robert.weidner@hsu-hh.de
}

The idea that humans cannot and do not want to be replaced by technology now and in future is followed by the desire for a balanced co-existence between human and technology. Therefore certain approaches to the development of assistance systems are needed based on individual and societal requirements at the same time. Since 2014 we organize the biennial trans-disciplinary conference entitled "technical support systems people really want" in Hamburg, Germany. Scientists, practitioners and potential users from different disciplines and sectors discussed, what kind of technical assistance people really appreciate and what kind of technical development approaches should therefore be pursued. This Minitrack provides the substantive continuation and at the same time serves as a stopover to the next conferences.

This Minitrack will discuss approaches for person- and task-adapted support systems for applications in daily and working life. In this context, theoretical and practical issues of the individual and social acceptance of technologies, their adaptability, design, usability and functionality will be considered. In 2016 the Minitrack focused hybrid-human support systems for work in industrial settings (e.g. exoskeletons in industrial production, textile industry) from both a theoretical and a practical standpoint. Presented research dealt with questions of desirability and acceptance of such systems based on empirical findings in different circumstances as well as optimization processes of colour selection in web design.

During the course of the 2017 Minitrack three papers will be presented:

This year's first paper "Situational Awareness and Systems for Driver-Assistance" by H. Alyamani and M. Kavakli tries to assess driver's situation awareness in order to design a driver-assistance program that helps drivers in unfamiliar traffic or driving situations and therefore may prevent vehicle accidents from happening.
Following that, "A Context-Aware Mobile Solution for Assisting Tourists in a Smart Environment" by F. Colace, S. Lemma, M. Lombardi, M. De Santo and M. Cassilo introduces an adaptive ContextAware app which was implemented in the city of Salerno. It helps tourists inside a touristic area through the collection of data from different sources like Internet of Things and Services.

In the last Paper of this session "Shop with me! Collaborative Information Searching and Shopping for Online Retail" by Y. Gao, M. Reddy and B. Jansen research on development of a collaborative search and shopping system for online retail tasks is presented. It aims at a better understanding of collaborative search tools and the way they improve information sharing among users.

In this field of research, we observe different paths of the development of physical and cognitive support systems that may also merge to complex systems soon. Hence, future research has to address the close connection of humans and technical systems. The advancements in the field will foster systems that are modular and that will be customizable in terms of individual needs. These systems will integrate data from several different sources by sensor systems. The will be analyzing situations by using artificial intelligence. Future Systems will also be able to identify intentions of their users. Because of the utilization of advanced materials, the next generation of assistance systems will be more comfortable and can be worn like clothes that fit the human body. 